\title{
Modification of Anion Exchange Membranes with Polystyrenesulfonic Acid
}

\author{
Koshi Kusumoto, Toshikatsu Sata, and Yukio Mizutani \\ Tokuyama Soda Co., Ltd., Tokuyama 745, Japan.
}

(Received August 13, 1975)

\begin{abstract}
KEY WORDS Anion Exchange Membrane/Organic Fouling / Sodium Dodecylbenzenesulfonate / Polystyrenesulfonic Acid /
\end{abstract}

As is well known, anion exchangers are susceptible to organic fouling, and many studies have been done with regard to the interactions between the anionic exchangers and organic foulants. ${ }^{1-3}$ For example, Small and Gardner have studied the poisoning of ion exchangers by surface active ions and effect of pretreating the ion exchangers with some polyelectrolytes. ${ }^{3}$ However, no details were given about the resistibility of the anion exchange membranes which were modified by dipping into an aqueous solution of polystyrenesulfonic acid (PSSA).

In order to understand the effect of the modification with PSSA, the fouling curves of the anion exchange membranes (Neosepta AV4T, Neosepta AF-4T, and Neosepta ACH-45T, from Tokuyama Soda Co., Ltd., prepared by the "paste method",4,5) have been investigated

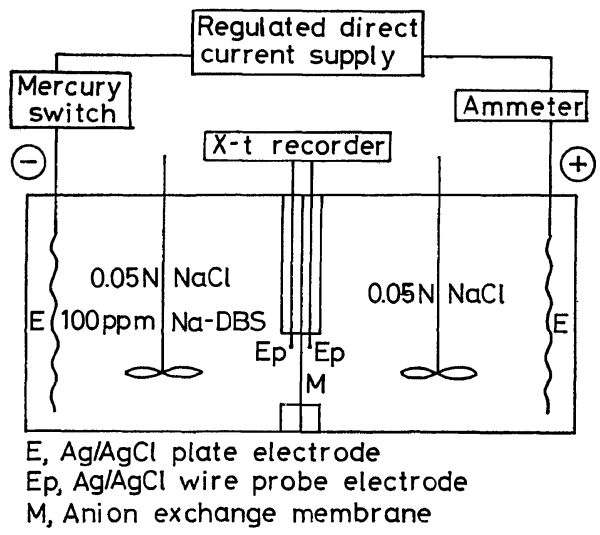

Figure 1. The apparatus: The effective area of the membrane was $10 \mathrm{~cm}^{2}$ and the volume of the solutions was $120 \mathrm{ml}$. by using sodium-dodecylbenzenesulfonate (NaDBS) as a foulant.

Here, PSSA was prepared by sulfonating a commercial polystyrene $(50 \mathrm{~g}$, Topolex $525-\mathrm{N} 100$ from Mitsui Toatsu Chemicals Inc.) with concentrated sulfuric acid $(500 \mathrm{~m} l)$ containing silver sulfate $(0.5 \mathrm{~g})$ at $100^{\circ} \mathrm{C}$ for $10 \mathrm{hr}$ with stirring; the resultant polymer was purified by means of dialysis. The anion exchange membranes were dipped in a $1-\%$ aqueous solution of PSSA and rapidly washed with water. The resistibility of the resultant anion exchange membranes was measured by using the apparatus shown in Figure 1: the change in the voltage across the membrane $\left(V_{\mathrm{m}}\right)$ was measured with stirring at

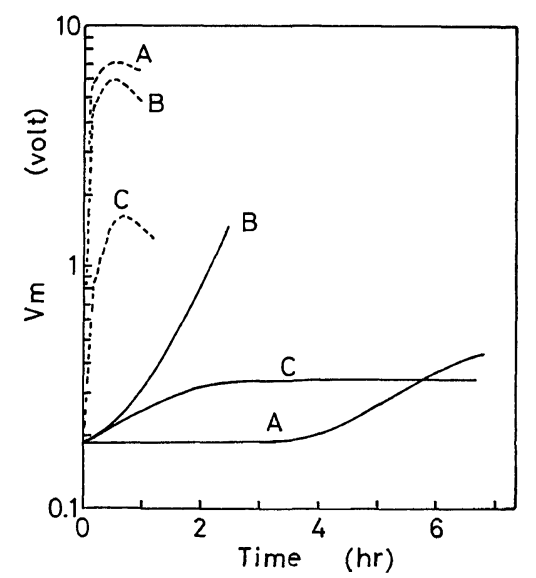

Figure 2. The fouling curves of the anion exchange membranes (the dotted lines are the controls): A, Neosepta ACH-45T; B, Neosepta AV-4T; C, Neosepta AF-4T. The treatment was at room temperature for $24 \mathrm{hr}$. 


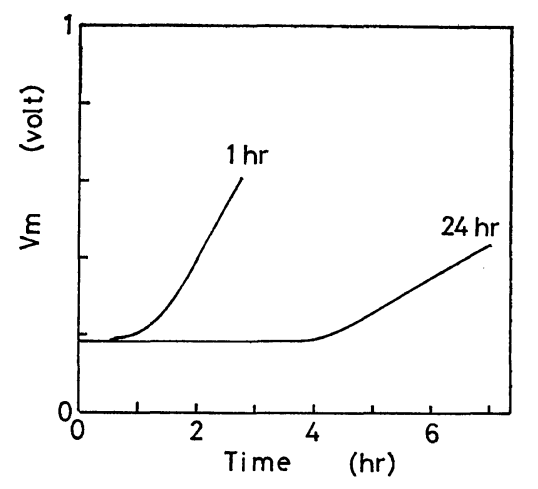

Figure 3. The effect of the treatment time on the fouling curve: The time shown in the figure shows the treatment time at room temperature.

$25^{\circ} \mathrm{C}$ by supplying direct current (current density, $2 \mathrm{~mA} / \mathrm{cm}^{2}$ ) and recorded with the aid of a $X-t$ recorder.

Figure 2 shows the fouling curves, that is, the change in the $V_{\mathrm{m}}$ value with time. It is interesting that the PSSA treatment did not cause any substantial increase in the $V_{\mathrm{m}}$ value. Although there was a question how the difference in the fouling curves of the modified membranes occurred, thd PSSA treatment was clearly effective for making the membranes resistant to the fouling. Its long term benefit, however, should be small, because the PSSA was not fixed onto the membrane surfaces. The anion exchange groups are fixed on the rigid matrixes of the membranes. On the other hand, PSSA is flexible, and some sulfonic acid groups of PSSA could be ion-exchangeable with the anion exchange groups on the membrane surface, but not all of the sulfonic acid groups. In other words, the formation of the polysalts on the membrane surfaces should be partially possible, so some amount of the sulfonic acid groups should remain free and show electrostatic repulsion against the foulant, Na-DBS.

The situation described above could be supported by the result shown in Figure 3: the longer the treatment time was, the more stable PSSA could be on the membrane surface and the more effective the treatment was. The results were not sufficiently quantitative and not perfectly reproducible, but could significantly suggest one possibility: if the sulfonic acid groups could appropriately be introduced onto the membrane surface, the resultant anion exchange membrane might be resistant to organic fouling for a long term.

\section{REFERENCES}

1. Saline Water Conversion Report, Office of Saline Water, (1969-1970), p 134 and p 143.

2. E. Korngold, F. De Körösy, R. Rahav, and M. F. Taboch, Desalination, 8, 195 (1970).

3. H. Small and R. C. Gardner, Office of Saline Water, Res. Develop. Prog. Rep., No. 565 (1970).

4. Y. Mizutani, R. Yamane, H. Ihara, and H. Motomura, Bull. Chem. Soc. Japan, 36, 361 (1963).

5. Y. Mizutani, R. Yamane, and H. Motomura, ibid., 38, 689 (1965). 